\title{
Hedonic and disgust taste perception in borderline personality disorder and depression
}

Gonzalo Arrondo, Graham K. Murray, Emma Hill, Bence Szalma, Krishna Yathiraj,

Chess Denman and Robert B. Dudas

\section{Summary}

Depression and borderline personality disorder (BPD) are both thought to be accompanied by alterations in the subjective experience of environmental rewards. We evaluated responses in women to sweet, bitter and neutral tastes (juice, quinine and water): 29 with depression, 17 with BPD and 27 healthy controls. The BPD group gave lower pleasantness and higher disgust ratings for quinine and juice compared with the control group; the depression group did not differ significantly from the control group. Juice disgust ratings were related to self-disgust in BPD, suggesting close links between abnormal sensory processing and self-identity in BPD.

\section{Declaration of interest}

None.

\section{Copyright and usage}

(c) The Royal College of Psychiatrists 2015. This is an open access article distributed under the terms of the creative Commons Attribution (CC BY) licence.
Reduced enjoyment of pleasant experiences and increased impact of negative emotions are observed clinically in depression and in borderline personality disorder (BPD). ${ }^{1}$ Surprisingly, previous laboratory studies do not support a link between depression and reduced pleasantness ratings to biological rewards, such as sucrose solutions (for example see Swiecicki et $a l^{2}$ ). However, patients with depression could be more sensitive to negative primary inputs. ${ }^{3}$ Few studies have used aversive stimuli in the evaluation of taste in depression, and no studies have been carried out to evaluate hedonic ratings of tastes in BPD in spite of clinically observable aberrant emotional processing and increased state and trait disgust. ${ }^{4}$ We hypothesised that patients with BPD and depression would differ from healthy controls in their pleasantness and disgust ratings to positive and especially negative taste stimuli.

\section{Method}

A total of 29 women with DSM-IV ${ }^{5}$ major depressive disorder, 17 women with DSM-IV BPD and 27 female healthy controls took part in the study, which was approved by the Cambridgeshire 4 National Health Service research ethics committee; all participants provided written informed consent. Additional details, including statistical data, are provided in the online supplement. Evaluation of taste consisted of participants taking a sip, but not swallowing, from a cup with $10 \mathrm{ml}$ of orange juice, quinine dihydrochloride at $0.006 \mathrm{~mol} / \mathrm{L}$ or water. Participants had to maintain the liquid in the mouth for $5 \mathrm{~s}$, rate the disgust and pleasantness produced using two visual scales (online Fig. DS1) and rinse their mouths with water. Order of liquids was counterbalanced across participants. Clinical evaluation was completed prior to the taste experiment. Statistical analysis (see online supplement for details) aimed to evaluate the association between taste disgust and disgust as measured using two clinical rating scales: the Self-Disgust Scale $(\mathrm{SDS})^{6}$ and the Disgust Scale Revised (DSR). ${ }^{7}$

\section{Results}

Overall differences between the three conditions in pleasantness and disgust ratings followed predictions across all participants. Quinine was highly unpleasant and disgusting, juice was highly pleasant and not disgusting and water was neither pleasant nor disgusting. Pleasantness and disgust ratings correlations were all significant. Regarding differences between groups, the BPD group rated both quinine and juice (but not water) as more unpleasant and disgusting than the control group, but no differences were found between the depression and control groups (all $P<0.05$; see online Tables DS8 and DS9, and Fig. 1). Increased self-disgust was significantly correlated (Spearman's $\rho=0.5$ ) with greater disgust ratings after the intake of orange juice in the BPD group (Table DS10). However, disgust propensity did not correlate with ratings in this group and all correlations were non-significant in the depression group.

\section{Discussion}

We found that in the BPD group there were abnormal pleasantness and disgust ratings after the intake of biological stimuli, whereas no differences between the depression and control groups were found. Our findings indicate that the hedonic experience of both positive and negative taste stimuli is negatively biased in BPD. This novel result is in line with clinical findings in the disorder, as people with BPD report more dysphoric and less positive affective cognitive states. ${ }^{1}$ Current diagnostic criteria for the disorder include affective instability, recurrent self-threatening behaviours and chronic feelings of emptiness, all of which could be related to negative perceptions of the environment.

The lack of evidence for a differential effect in the case of depression is also in line with most of the existing literature on enjoyment of pleasant tastes; ${ }^{2}$ however, our study also shows that there were no differences between the depression group and the control group in evaluation of a disgusting taste. A limitation of our study and the prior depression studies is that sample sizes were small; hence, either there is no true difference in the ratings for chemosensory stimuli between people with depression and controls or the effect is small, suggesting that the basis of anhedonia reported in depression is complex. For example, it could be that clinically observed anhedonia in depression is primarily related to social anhedonia. Alternatively, clinical assessments of anhedonia may confound motivational, anticipatory and mnemonic aspects of enjoyment with consummatory 'in the moment' pleasure; the latter is assayed by our laboratory taste task and may be comparatively intact in depression. ${ }^{8}$

In the BPD group, questionnaire-measured self-disgust, but not disgust propensity, correlated with laboratory-rated disgust 
(a)

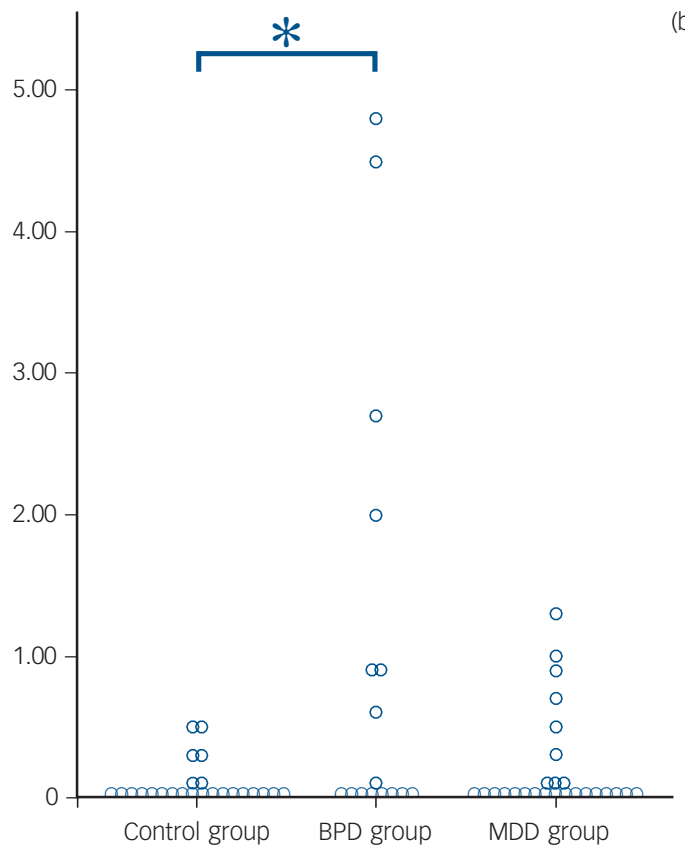

(b)

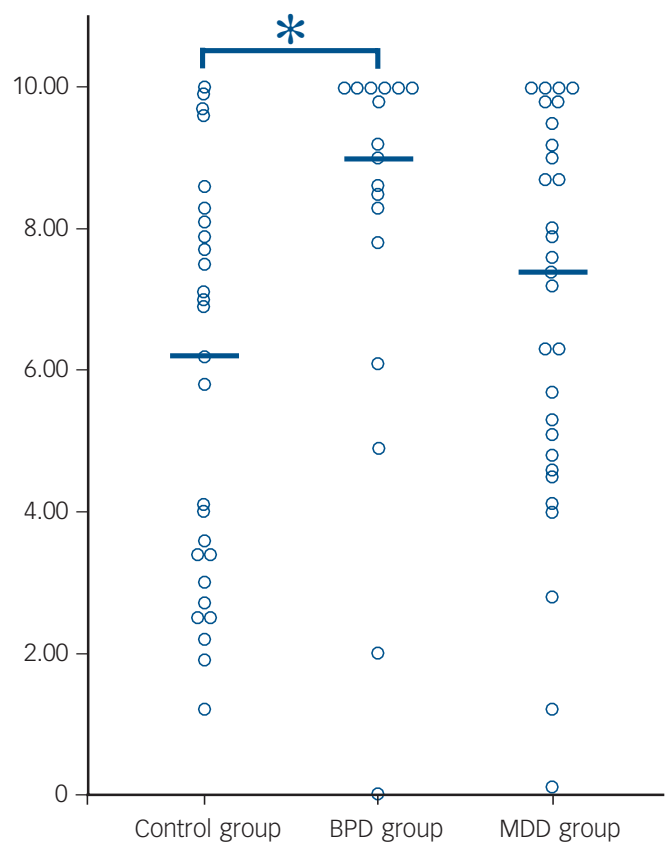

Fig. 1 Scatter plot with the (a) juice and (b) quinine disgust ratings stratified by group.

The horizontal brackets with asterisks indicate significant group differences and the horizontal lines group medians. BPD, borderline personality disorder; MDD, major depressive disorder.

to juice stimuli. Self-disgust indicates a context-free negative evaluation of the self (shame feelings) and also negative views about one's actions (guilt), ${ }^{6}$ and it is greatly enhanced in BPD. Self-disgust may be felt as an embodied experience instead of an abstract sensation, to the point of producing negative physical sensations such as nausea, and is often triggered by external events. ${ }^{9}$ Our finding of an association between self-disgust and juice-disgust indicates close links between sensory processing and self-identity in BPD, and may suggest that basic physiological disturbances play a role in the origins of self-disgust in this disorder. Previous research indicates that self-disgust is correlated with overall symptom severity in BPD and eating disorders, ${ }^{10}$ which could indicate similar mechanisms within the two disorders. ${ }^{11}$ We speculate that in BPD self-disgust is so heightened that it may impair the enjoyment of stimuli that are ordinarily considered as pleasant. Alternatively, a fundamental abnormality in processing external sensory stimuli may contribute to a negative sense of self in BPD. The less positive ratings could also be related to other group-specific factors, such as an increased history of trauma in the BPD group. ${ }^{4,12}$ Our results emphasise the significance of disgust - both of the self and of external stimuli - in BPD, and highlight a role for assessment of disgust in the diagnosis and management of this condition. ${ }^{4}$

Gonzalo Arrondo, PhD, Department of Psychiatry, University of Cambridge Cambridge; Graham K. Murray, MD, PhD, MRCPsych, Department of Psychiatry, University of Cambridge, Cambridgeshire and Peterborough NHS Foundation Trus and Behavioural and Clinical Neuroscience Institute, University of Cambridge Cambridge; Emma Hill, PhD, Cambridgeshire and Peterborough NHS Foundation Trust, Cambridge; Bence Szalma, BSc, Department of Psychiatry, University of Cambridge, Cambridge; Krishna Yathiraj, MRCPSych, Department of Psychiatry, Cambridge, Cambridge; Krish a Yathiraj, MrcPsych, Department of Psychiatry, University of Cambridge, Cambridge and Norfolk and Suffolk NHS Foundation Trust, Norwich; Chess Denman, MRCPsych, Cambridgeshire and Peterborough NHS Foundation Trust, Cambridge; Robert B. Dudas, MD, PhD, MRCPsych, Department of Psychiatry, University of Cambridge, Cambridgeshire and Peterborough NHS Foundation Trust, Behavioural and Clinical Neuroscience Institute, University of Cambridge, Cambridge, and Psychiatric Liaison Service, Ipswich Hospital, Norfolk and Suffolk NHS Foundation Trust, Norwich, UK

Correspondence: Graham K. Murray, Department of Psychiatry, University of Cambridge, Herchel Smith Building, Forvie Site, Robinson Way, Cambridge Biomedical Campus, Cambridge CB2 OSZ, UK. Email: gm285@cam.ac.uk

First received 20 Apr 2014, final revision 30 Oct 2014, accepted 7 Nov 2014

\section{Funding}

Supported by the Wellcome Trust (093875/Z/10Z and 09814/Z/11), Medical Research Council (G0701911 and G1000183), Isaac Newton Trust, Cambridgeshire and Peterborough NHS Foundation Trust, Talisman Trust, University of Cambridge and the NIHR Cambridge Biomedical Research Centre.

\section{References}

1 Reed LI, Fitzmaurice G, Zanarini MC. The course of positive affective and cognitive states in borderline personality disorder: a 10-year follow-up study. Personal Ment Health 2012; 6: 281-91.

2 Swiecicki L, Zatorski P, Bzinkowska D, Sienkiewicz-Jarosz H, Szyndler J, Scinska A. Gustatory and olfactory function in patients with unipolar and bipola depression. Prog Neuropsychopharmacol Biol Psychiatry 2009; 33: 827-34.

3 Leppanen JM. Emotional information processing in mood disorders: a review of behavioral and neuroimaging findings. Curr Opin Psychiatry 2006; 19: 34-9.

4 Schienle A, Haas-Krammer A, Schoggl H, Kapfhammer HP, Ille R. Altered state and trait disgust in borderline personality disorder. J Nerv Ment Dis 2013; 201: 105-8.

5 American Psychiatric Association. Diagnostic and Statistical Manual of Mental Disorders (4th edn) (DSM-IV). APA, 1994.

6 Overton PG, Markland FE, Taggart HS, Bagshaw GL, Simpson J. Self-disgust mediates the relationship between dysfunctional cognitions and depressive symptomatology. Emotion 2008; 8: 379-85.

7 Olatunji BO, Williams NL, Tolin DF, Abramowitz JS, Sawchuk CN, Lohr JM, et al. The Disgust Scale: item analysis, factor structure, and suggestions for refinement. Psychol Assess 2007; 19: 281-97.

8 Dillon DG, Rosso IM, Pechtel P, Killgore WD, Rauch SL, Pizzagalli DA. Peril and pleasure: an rdoc-inspired examination of threat responses and reward processing in anxiety and depression. Depress Anxiety 2014; 31: 233-49.

9 Powell PA, Overton PG, Simpson J. The revolting self: an interpretative phenomenological analysis of the experience of self-disgust in females with depressive symptoms. J Clin Psychol 2014; 70: 562-78.

10 Ille R, Schoggl H, Kapfhammer HP, Arendasy M, Sommer M, Schienle A Self-disgust in mental disorders - symptom-related or disorder-specific? Compr Psychiatry 2014; 55: 938-43.

11 Zanarini MC, Reichman CA, Frankenburg FR, Reich DB, Fitzmaurice G. The course of eating disorders in patients with borderline personality disorder: a 10-year follow-up study. Int J Eat Disord 2010; 43: 226-32.

12 Rusch N, Schulz D, valerius G, Steil R, Bohus M, Schmahl C. Disgust and implicit self-concept in women with borderline personality disorder and posttraumatic stress disorder. Eur Arch Psychiatry Clin Neurosci 2011; 261 369-76. 